\title{
Transposition didactique et rapports aux savoirs :

\author{
l'exemple des enseignements \\ de questions économiques et sociales, \\ socialement vives
}

Alain Legardez

Nous proposons une analyse des problèmes liés à l'enseignement des questions économiques et sociales qui s'inscrit dans la tension entre la reconnaissance générique du concept de transposition didactique et l'étude des spécificités de la gestion des rapports aux savoirs dans les enseignements de "questions socialement vives ". Le bien fondé de l'utilisation de la théorie de la transposition didactique est discuté à partir de l'exemple de la discipline des sciences économiques et sociales (SES) où elle a donné lieu à de vives controverses. Une analyse en termes de construction de distances entre différents savoirs est proposée pour contribuer à l'étude de la problématicité des enseignements, déjà pointée par la théorie de la transposition didactique. Les illustrations sont prises dans des travaux récents menés en didactique des disciplines concernées.

Mots-clés : didactique comparée, questions socialement vives, rapports aux savoirs, sciences économiques et sociales, transposition didactique.

'une des voies du développement de la didactique aussi que leurs spécificités (1) appellent des analyses des

disciplines semble désormais passer par des fines faisant également appel à d'autres outils théo-

travaux de didactique comparée (Johsua, 2001 ; Mercier et al., 2002) dont l'un des objectifs est d'étudier la pertinence de l'utilisation des concepts de la théorie didactique dans le champ des différentes didactiques disciplinaires. Les travaux que nous menons dans le champ des enseignements économiques, sociologiques, juridiques et de gestion cherchent notamment à montrer que les principaux concepts de la théorie didactique peuvent être convoqués pour étudier les questions liées à ces enseignements, mais riques (Legardez, 2001).

Nous tenterons de montrer que les questions que pose la théorie de la transposition didactique sont bien incontournables dans le champ de la recherche en didactique, mais que l'utilisation du concept nécessite des spécifications dans la perspective d'études de la gestion des rapports aux savoirs, notamment dans 
l'enseignement d'objets scolaires liés à des questions " économiques et sociales, socialement vives ». Pour illustrer notre utilisation du concept-clé de la transposition didactique (TD) dans le domaine de la didactique des enseignements économiques et sociaux, nous proposons comme objet cible la discipline scolaire des "Sciences Économiques et Sociales » (SES) de la filière "Économique et Sociale » (ES) des lycées français.

\section{TRANSPOSITION DIDACTIQUE ET ENSEIGNEMENTS ÉCONOMIQUES ET SOCIAUX}

La question de la nature et de l'origine des contenus des savoirs scolaires est cruciale pour toute analyse didactique disciplinaire. Les disciplines concernées par les enseignements économiques et sociaux ne font pas exception, bien que la question se pose de manière parfois plus polémique que pour d'autres disciplines (2). Les débats menés depuis deux décennies pour critiquer l'utilisation de la théorie de la transposition didactique en SES en sont un bon exemple (3).

\section{Les critiques à la théorie de la transposition didactique}

\section{Le refus de la théorie de la TD en SES}

Dans ses travaux sur les SES, l'objectif d'É. Chatel est de décrire le processus en acte d'enseignementapprentissage, au sens large puisqu'il inclut le passage des savoirs de référence aux savoirs enseignés (Chatel 1995, 2001). Elle rejette essentiellement la référence unique au savoir savant, issue de la théorie de Chevallard (1991). En fait, sa critique des références des enseignements de SES comporte deux aspects. D'une part l'appel à des références complémentaires - les « pratiques sociales de référence » de J.-L. Martinand -, apparaît comme un précieux contrepoids aux savoirs-savants. D'autre part l'auteur signale le double problème de l'existence de savoirs savants pluriels en sciences sociales et de la recomposition des savoirs de la discipline que l'université et la recherche séparent le plus souvent. En dernière analyse, l'inadaptation de la théorie de la TD aux SES tiendrait avant tout à la spécificité de ses contenus : "Les contenus donnés à l'enseignement des SES constituent donc une option, celle de la convergence des sciences sociales, dans un débat particulièrement épineux entre l'économie et les autres sciences sociales, puisqu'il touche à l'objet même de l'économie. "(Chatel 1995, p. 12). Se réclamant de l'affirmation d'Y. Chevallard selon laquelle il conviendrait que le savoir savant et le savoir enseigné "se ressemblent suffisamment ", l'auteur en déduit qu'il s'agit là d'une normativité qui remettrait en cause la liberté pédagogique et ne permettrait guère d'expliquer l'histoire originale des SES. Elle considère que le concept de "transformation des savoirs " qu'elle propose lui permet d'analyser ce qui se passe réellement dans le processus de production d'enseignable. Cette critique laisse néanmoins ouverte la question de la nature et du processus de légitimation de la discipline scolaire des SES. La légitimité se fondet-elle sur l'existence même d'une discipline scolaire nouvelle dans le système éducatif français (4), sur un « projet fondateur » (aux deux sens du terme : initial et légitimant) ou sur un nécessaire rapport à des savoirs de référence?

E. Triby s'est intéressé directement à la question de la transposition didactique, donc au problème des savoirs de référence pour les SES, et notamment dans leurs aspects les plus économiques (Triby 1995, 1997). Sa réflexion s'appuie sur une conception très relativiste des sciences sociales, assez largement répandue chez les professeurs de SES. Selon cet auteur, les théories économiques sont des productions sociales, donc des productions idéologiques qui ne sont pas différentes par nature des savoirs (des représentations) des élèves. La critique de la théorie de la TD est directe : "Chez Y. Chevallard, (la notion de TD) se réduit à l'affirmation que le savoir enseigné tire essentiellement sa légitimité de sa plus grande "proximité" par rapport à un savoir savant de référence, et de sa plus grande distance par rapport au "savoir spontané", vulgaire, le "savoir des parents". La solidité apparente de sa construction a fini par installer la TD dans une fonction de modèle applicable à toutes les espèces de disciplines scolaires. En SES pourtant, elle ne va pas de soi, c'est le moins qu'on puisse dire. " (Triby 1995, p. 119). La nature des savoirs savants de référence, - et en particulier leur caractère pluriparadigmatique -, et la « confusion » de leur identité les rendraient inaptes à la transposition. C'est cette version des travaux d'E. Triby qu'utilisent couramment les adversaires de la TD, et plus généralement de la référence à des savoirs savants.

\section{Une résistible évolution}

É. Chatel reconnaît désormais que le concept de TD " met en effet l'accent sur ce qui sépare le régime du savoir didactisé de l'école, de la création de 
connaissance dans l'ordre de la science... ॥ ; mais elle regrette "que cette articulation (soit pensée) seulement en termes de légitimité "(Chatel 1999, p. 23), alors que la notion "d'enseignabilité » lui semble incontournable pour les SES. E. Triby souhaite aller " à la recherche d'une identité épistémologique par rapport aux sciences sociales de référence, d'une identité didactique et pédagogique au sein du système d'enseignement, ce qui implique une analyse de la transposition des savoirs de référence ainsi qu'une analyse des processus de socialisation des savoirs sociaux. " (Triby 1997, p. 319). II n'y a donc plus rejet de la théorie de la TD, mais réflexion sur les spécificités de ces processus pour la discipline scolaire des SES. Néanmoins, le débat interne à la discipline est résumé comme celui entre "la pédagogie inductive... vue comme ayant une portée d'éducation à l'autonomie, et opposée à une définition de la discipline en termes de transposition didactique des sciences économiques d'une part, de la sociologie d'autre part » (Chatel 2002, p. 32).

L'évolution est progressive et elle se heurte à de fortes résistances, ce qui montre que certaines des questions soulevées par la théorie de la TD sont bien incontournables, en SES comme ailleurs, mais aussi que la critique principale reste liée au soupçon de " normativité 》 qui correspond à une lecture très contextualisée de la théorie de la TD, celle d'une défense de la spécificité d'une discipline scolaire pensée par certains comme partiellement « hors norme $»$.

\section{Le caractère heuristique de la théorie de la transposition didactique}

$\mathrm{Si}$ on accorde aux discours à visée scientifique (Granger, 1993) sur le social le même statut qu'aux discours sur la nature, le travail transpositif s'impose pour les enseignements économiques et sociaux, au même titre que pour les disciplines scientifiques. II importe alors d'en étudier les spécificités.

\section{Transposition didactique et pratiques sociales de référence}

Le concept de «pratiques sociales de référence » proposé par J.-L. Martinand (1986) est souvent pris comme une alternative à celui de la TD ; or, il n'est pas à l'origine une critique directe de celui de transposition didactique. Ce que craint cet auteur, c'est un " usage dogmatique " du concept de TD. C'est pourquoi, sans nier l'intérêt du concept, il dit utiliser selon les thèmes étudiés, les notions " de transposition restreinte (entre savoir savant et savoir enseigné), de transposition générale (entre pratiques de référence et activités scolaires)... de reformulation ou de transcodage ... de construction ou de composition sous contraintes " (Martinand 2001, p. 21-22). À notre sens, l'auteur attire l'attention sur trois questions cruciales qui interpellent les didactiques disciplinaires. D'une part, il insiste sur la nécessité de penser la question décisive qui reste celle de la référence des objets d'enseignements scolaires, sans sombrer dans le mythe de l'autoréférentialité, alors qu'une tendance forte consiste à nier la question des rapports aux références pour se réfugier dans une spécificité, comme celle des " savoirs-SES ». D'autre part, il pointe le danger qu'il y aurait à galvauder le terme de "savoirs " sans en avoir suffisamment pensé le contenu, rejoignant la préoccupation de Chevallard qui précise "qu'on ne parle jamais de savoir que comme métonymie. La partie - le savoir - désigne un tout qui inclut aussi domaine de réalité et pratique sociale. » (Chevallard 1994, p. 176). Enfin, il signale que les pratiques de référence " actuelles et évolutives ou virtuelles, doivent être objectivées, et en vérité modélisées. 》 (Martinand 2001, p. 20), pointant ainsi la nécessaire mise à distance des références dans la construction des savoirs scolaires, donc leur " transposition ». Cette insistance sur la nécessité "de penser et analyser les écarts entre activités scolaires et pratiques prises comme référence " (id., p. 19), nous semble en effet plus s'inscrire dans une généralisation de la problématique de la TD que dans une critique de son caractère heuristique.

\section{Transposition, choix axiologiques et savoirs sociaux}

De nombreux enseignants d'autres disciplines ont eu une réaction analogue à celle des enseignants de SES, et ont prétendu que la validité de la TD ne dépassait pas le domaine des didactiques scientifiques, ... ou même de la didactique des mathématiques, ce qui a donné lieu à de nombreux débats (Arsac et al. 1994, Caillot \& Raisky et al. 1996, Petitjean et al. 1998). Certains ont critiqué l'usage du concept de TD pour les disciplines comme les SES où le domaine des valeurs est évident. En effet, dans leur présentation des programmes, les concepteurs fixent les finalités des savoirs à enseigner qui sont les objectifs généraux d'un enseignement ; par exemple : " faire en sorte que les élèves deviennent des citoyens autonomes et responsables ». Ces finalités ne sont pas immuables ; elles constituent un choix qui renvoie à un système de valeurs dans un contexte socioéconomique, politique et culturel donné. M. Develay (1995, p. 215) parle de "choix axiologiques" 
pour qualifier ces choix qu'opèrent les concepteurs de programmes. Le passage des savoirs savants et des pratiques sociales ou professionnelles aux savoirs enseignés se fait donc (aussi) selon des choix axiologiques. Le choix des savoirs à enseigner n'est pas unique ; il y a donc un large éventail des possibles pour les concepteurs de programmes.

Loin de contredire la théorie de la TD, il nous semble qu'il s'agit là d'une question classique de la transposition didactique externe. En effet, cela ne signifie pas que l'enseignant est strictement contraint par ces choix. Du fait de la pluralité des possibles se dessine l'espace de la liberté pédagogique : l'enseignant effectue ses propres choix pédagogiques et en est responsable ; ce travail est alors caractéristique de la transposition didactique interne. Dans une perspective didactique, il est donc important d'étudier les choix axiologiques des concepteurs de programmes et les propres choix des enseignants, en SES comme ailleurs. Comme le signale S. Johsua : "Le concept de transposition didactique nous conduit à admettre l'artificialité constitutive des actes didactiques. II nous ferme la fallacieuse "voie naturelle" (importer la "vie" dans l'école), mais nous ouvre un fantastique espace de liberté, puisqu'il fonde en droit la possibilité d'exister de plusieurs constructions artificielles concurrentes. " (Johsua 1996, p. 23)

\section{Légitimité et normalisation}

Le refus de la TD ne proviendrait-il pas alors de la crainte de la mise à jour d'un " terrible secret " (Chevallard) : celui d'une autolégitimation des contenus par les enseignants de SES. La question de la légitimité n'a pas à être posée pour ceux qui défendent l'idée d'un « savoir-SES » qui possèderait ses propres normes de scientificité et qui serait autolégitimé. Si l'on conteste le statut de scientificité aux discours académiques sur la société en les considérant comme des discours idéologiques au même titre que d'autres discours sur le social, la transposition peut être niée puisqu'il n'y aurait rien à transposer. Les discours sur le social seront mis sur le même plan que les représentations des élèves et traités comme tels dans la construction des savoirs dans la classe. Certains auteurs aboutissent ainsi à une double négation de la transposition didactique en SES : d'une part, les savoirs scolaires ne s'autorisent que d'eux-mêmes et refusent donc tout renvoi à quelque savoir de référence que ce soit ; et d'autre part, ils se définissent par la représentation que les enseignants ont de ce qui est acceptable par les élèves. En fait, les contenus de l'enseignement importeraient moins que la méthode. Les discours de ces enseignants rabattent la spécificité des SES sur les «méthodes actives 》 dont ils seraient les spécialistes, et le " savoir SES 》 se définirait alors comme un savoir pratique socialisé, produit dans l'interaction pédagogique en référence à des postures pédagogiques plutôt qu'à de véritables savoirs de référence. Pourtant, l'histoire des SES montre une tendance longue à une normalisation, au renforcement des références explicites à des savoirs savants (en économie, sociologie et sciences politiques) (Chatel, 1990 ; Combemale et al., 1995 ; Alpe, 1995 ; Beitone \& Legardez, 1995 ; Legardez, 2001). La filière ES deviendrait une filière comme les autres, qui s'inscrit dans une logique hiérarchique de noblesse des filières (Cazals, 1998) (5). On voit par là que si les SES ont bien renforcé leur stabilité écologique, c'est sans doute au prix de la renonciation à leur spécificité mythique (la « mission sociale » originelle) (6).

La TD est bien une « théorie » : " ... ce n'est pas une description, une image plus ou moins imparfaite de la réalité théorisée. Ce n'est qu'une machine à produire des connaissances d'un certain type à propos de la réalité soumise à la question " (Chevallard 1994, p. 164). La question posée est celle de "la problématicité des savoirs " (Chevallard 1997b), des " écarts " entre les références et les savoirs scolaires; elle nous semble donc incontournable.

\section{GESTION DES RAPPORTS AUX SAVOIRS DANS LES ENSEIGNEMENTS LIÉS À DES QUESTIONS SOCIALEMENT VIVES}

En nous inspirant de ce cadre théorique, nous proposons de préciser notre problématique en termes de gestion des distances aux différents savoirs pour l'étude des questions économiques et sociales, notamment lorsqu'elles sont « socialement vives » (7).

\section{Les spécificités de l'étude de questions économiques et sociales, socialement vives}

Les "questions socialement vives " dans le champ scolaire

Nous appellerons «question socialement vive » une question qui possède les caractéristiques suivantes :

- $\quad$ Elle est vive dans la société : une telle question interpelle les pratiques sociales des acteurs scolaires (dans et hors de l'institution) et renvoie à leurs 
représentations sociales ; elle est considérée comme un enjeu par la société (globalement ou dans certaines de ses composantes) et suscite des débats ; elle fait l'objet d'un traitement médiatique tel que la majorité des acteurs scolaires en ont connaissance. Sa production sociale dans la société la rend "vive » dans un premier sens.

- Elle est vive dans les savoirs de référence : des controverses sont courantes entre spécialistes des champs disciplinaires ou entre les experts des champs professionnels. Dans la plupart de ces savoirs disciplinaires qui renvoient à des sciences sociales et humaines, plusieurs paradigmes sont en concurrence. Sa production sociale dans des milieux scientifiques, sociaux ou professionnels la rend "vive » dans un second sens.

II ne s'agit pas toujours à proprement parler de questions d'actualité : elles peuvent demeurer vives pendant de longues périodes, avec une intensité variable dans le temps et dans les différentes composantes de la société. C'est ainsi que la question des revenus (de leur origine, de leur répartition et de leur redistribution), la question de l'emploi et du chômage, la question de l'entreprise (sa fonction économique et sociale, son gouvernement), la question du risque et de l'incertitude en économie, de la mondialisation... sont des antiennes des débats économiques, aussi bien au sein du débat scientifique (entre théoriciens libéraux et interventionnistes, par exemple) que dans les débats sociaux et politiques. Des questions socialement vives ne sont pas toujours présentes dans les curricula sous forme de «questions ». II s'agit le plus souvent d'objets d'enseignement scolaire qui renvoient à des questions vives dans les références et/ou dans les savoirs sociaux, mais qui ne se présentent pas sous une forme scolaire problématisée. Les processus de didactisation ont pu leur faire perdre l'apparence d'une question sociale pour en faire un objet d'enseignement scolaire " neutralisé ». Des objets inscrits dans les programmes ou référentiels des enseignements économiques français peuvent ainsi être déproblématisés et être proposés aux enseignants puis aux élèves sous la forme de savoirs scolaires déclaratifs, sans réels enjeux d'apprentissage. C'est ainsi que l'on peut repérer (à partir d'une analyse des programmes, des manuels et d'enquêtes auprès d'enseignants) un dégradé de problématisation de l'enseignement du thème des revenus en fonction de la filière : éléments de problématisation réelle pour la filière ES (en classe de Seconde), moindre pour la filière Sciences et Technologies Tertiaires (en classe de
Première) et faible pour les filières professionnelles tertiaires (en classe de baccalauréat professionnel). Par exemple, les tâches les plus basiques de simple restitution de faits et données présents dans des textes et tableaux représentent plus de $50 \%$ de l'ensemble des tâches proposées dans les manuels des lycées professionnels, environ $35 \%$ dans ceux de STT et $25 \%$ dans ceux de SES (8).

Une analyse en termes de savoirs et de rapports aux savoirs

La spécificité d'une problématique didactique, c'est d'abord de situer l'analyse par rapport aux savoirs. Nos travaux s'inscrivent aussi dans la lignée des analyses des rapports aux savoirs (Charlot, 1997 ; CharlotBautier-Rochex, 1992 et 2000 ; Terrisse, 2001) et aux différents " genres » de savoirs (Chevallard 1997b). La question des différents savoirs ne peut donc se concevoir en dehors d'une étude de leurs rapports, ni d'une réflexion de type épistémologique. Au sens large, on peut parler de trois "épistémologies » ou de trois systèmes de rapports aux savoirs qui coexistent dans les processus de production de savoirs scolaires. L'épistémologie savante des savoirs de référence (souvent objet de débats au sein des communautés scientifiques en sciences sociales) peut être importée comme telle dans les savoirs à enseigner ou modifiée par la noosphère (9) dans le cadre de la TD externe. L'épistémologie de l'enseignant est une construction issue partiellement de son histoire privée qui peut être notablement différente de l'épistémologie institutionnelle et de celle de la noosphère ; souvent fortement marquée par l'acculturation disciplinaire, c'est une composante de la TD interne. L'épistémologie de l'élève est l'ensemble des représentations qu'il s'est construit sur le statut des discours à vocation scientifique sur le social (où se mêlent opinions et connaissances), sur le statut des disciplines (économie, sociologie...), sur ses rapports individuels et collectifs à l'École et au Savoir ainsi qu'aux différents savoirs (Léziart, 1995).

On conçoit la difficulté à gérer ces conflits potentiels de rapports aux savoirs que va devoir assumer l'enseignant. La recherche en didactique ne peut donc faire l'économie d'en analyser finement les entrelacs.

\section{Production de savoirs scolaires et gestion des rapports aux savoirs}

\section{La construction des distances aux savoirs}

Notre analyse de l'enseignement de questions socialement vives s'appuie sur la distinction entre trois 
genres de savoirs théoriquement bien distincts : les « savoirs de référence ", les "savoirs sociaux », et les " savoirs scolaires »:

Les savoirs de références sont essentiellement -pour des disciplines générales comme les SES - des savoirs savants et des pratiques sociales (10).

- Les savoirs sociaux sont ceux des acteurs del'école (des élèves, mais aussi des parents et des enseignants) ; ces savoirs construits hors de l'école deviennent des " savoirs préalables " lorsqu'ils sont importés dans l'école. Ils peuvent être partiellement constitués de véritables représentations sociales (Vergès, 1989) et ils peuvent inclure des éléments de savoirs scolaires antérieurement appris et partiellement exportés vers les savoirs sociaux; on peut alors parler de « systèmes de représentationsconnaissances " (Beitone \& Legardez, 1995).

- Enfin, les savoirs scolaires sont un troisième gen-re de savoirs. Ces savoirs scolaires sont construits d'une part en relation avec les deux autres genres de savoirs et d'autre part avec les autres sous-ensembles de savoirs scolaires : savoirs institutionnels de référence (programmes, référentiels, sujets d'examen...), " savoirs intermédiaires " (manuels, revues professionnelles ou de vulgarisation...).

À chaque moment du processus de production des objets d'enseignements scolaires, le concepteur de programmes (l'expert dans la " sphère de l'expertise » processus de TD externe -) et l'enseignant (dans la « sphère de l'enseignement " - processus de TD interne -) tentent de gérer au mieux leurs rapports aux savoirs. C'est ainsi que nous avons pu analyser la production des savoirs scolaires sur les revenus comme un double processus de déproblématisationreproblématisation : essentiellement des savoirs de référence aux savoirs scolaires institutionnalisés (programmes) dans la sphère de l'expertise dans un premier temps, des savoirs scolaires institutionnels aux savoirs enseignés dans la sphère de l'enseignement dans un second temps, le plus souvent via les savoirs intermédiaires que sont les manuels (Legardez et al., 2002).

\section{La gestion des rapports aux savoirs}

Si l'on se place du point de vue de l'enseignant, il nous semble que cette analyse permet de préciser quelques questionnements :

- Sur les « degrés de vivacité »: on peut penser que l'enseignant aura d'autant plus de problèmes spécifiques à gérer que l'objet scolaire concerné renverra à des questions particulièrement « vives » dans les deux autres genres de savoirs. C'est ainsi qu'à certaines périodes les questions comme celles des revenus ou du chômage sont des questions vives dans la société ; chaque sujet social se trouve directement ou indirectement concerné, alors qu'elle est aussi l'objet de vives controverses entre économistes et entre sociologues. De même, le degré de vivacité de la question de l'entreprise peut varier en fonction des débats en cours dans la société (ou dans certains de ses sous-ensembles), dans les sciences et les pratiques de références... mais aussi en fonction de son statut dans les savoirs à enseigner : objet d'enseignement parmi d'autres pour les SES, mais méta-objet de l'ensemble des programmes et référentiels en économie-gestion (Legardez \& Lebatteux, 2002). Quant à la question du risque et de l'incertitude en économie, elle peut être avivée par des évènements climatiques, politiques, économiques, sociaux... alors que les débats théoriques font florès entre les économistes, par exemple entre ceux qui postulent que l'incertitude peut être anticipée et calculée (néoclassiques) et d'autres pour lesquels l'incertitude est radicale, consubstantielle à la fondamentale méconnaissance du futur (keynésiens). Pour gérer cette double vivacité dans sa classe, l'enseignant aurait aussi à tenir compte des savoirs préalables des élèves qui pensent que : "l'incertitude en économie de marché est normale et que le risque est un choix assumé par les seuls petits entrepreneurs " (Legardez, 2004a) ; ces savoirs peuvent en effet faire directement obstacle à des apprentissages liés aux théories économiques néoclassiques.

- Sur les « processus de légitimation 》: l'enseignant va chercher à légitimer au mieux les contenus de son enseignement. Cette légitimation sera d'une part " externe » aux savoirs scolaires : par rapport à ce que l'enseignant peut appréhender de la demande sociale, par rapport aux savoirs savants et aux pratiques sociales de référence, et parfois aussi en fonction de ce qu'il se représente des savoirs sociaux des élèves. Des travaux menés sur l'enseignement de nombreux objets d'enseignement scolaire liés à des questions économiques et sociales vives montrent qu'une inadéquation partielle entre ces anticipations et les savoirs préalables des élèves peut poser de redoutables problèmes dans la relation didactique. Par exemple, une de nos premières études sur l'enseignement de la monnaie a montré que des enseignants de SES anticipaient que $75 \%$ de leurs élèves pensent que la valeur de la monnaie repose sur des métaux précieux, alors qu'en réalité ils n'étaient que $25 \% \ldots$ et que cette représentation était beaucoup 
moins résistante que ne le pensaient les enseignants (Beitone \& Legardez, 1997). Plus récemment une étude sur l'enseignement de l'entreprise montrait que l'inadéquation peut perdurer : des élèves de lycées professionnels interrogés en fin d'année en milieu scolaire assimilaient " production » et " productivité ", ce dernier étant " un mot de prof ». Plus généralement, ils tenaient un discours en bonne part d'un " autre monde " que celui de leurs enseignants dont le discours reste peu légitime à leurs yeux (Legardez \& Lebatteux, 2002). La légitimation sera construite d'autre part en « interne », par rapport aux savoirs scolaires de référence, voire par rapport aux «savoirs intermédiaires " (manuels). Nos études sur des thèmes économiques comme les revenus semblent montrer que la diversité des possibles dans le cadre de ce que les enseignants revendiquent comme leur liberté pédagogique est fortement réduite dans la réalité de leur travail, essentiellement par l'intériorisation de normes du corps d'enseignants auquel ils appartiennent et par le rôle crucial des manuels, aussi bien dans la circulation (ou la sédimentation) des savoirs que dans la reproduction des cultures disciplinaires. Les enseignants semblent ainsi déléguer en bonne partie le travail de reproblématisation aux auteurs de manuels (Legardez et al., 2002).

- Sur le double processus de " reproblématisation ": on peut faire l'hypothèse que l'enseignant cherche à construire en permanence ce qu'il considère comme la " bonne distance » (11) par rapport aux différents genres de savoirs. II est alors conduit à une double reproblématisation : d'abord celle qui concerne les savoirs qu'il cherche à faire circuler (ou non) entre les genres de savoirs et son enseignement ; ensuite, celle qui l'amène à " activer " ou à “ 2004b).

Des savoirs issus des sciences sociales ou des pratiques sociales prennent ainsi « forme scolaire ", sont transposés, re- ou dé-problématisés en savoirs à enseigner ... on aura reconnu une adaptation à des questions socialement vives du double processus de la transposition didactique externe et interne.

\section{CONCLUSION}

La question des rapports aux différents savoirs (savoirs et pratiques de référence, savoirs sociaux des élèves, savoirs scolaires) peut s'analyser à la fois comme une spécification et une généralisation de la question de la transposition didactique, question qui s'inscrit dans une culture qui lui donne sens et à laquelle elle participe ; elle apparaît incontournable, notamment dans les didactiques des enseignements économiques et sociaux. Et l'une des principales spécificités de la réflexion didactique sur l'enseignement d'objets scolaires liés à des "questions économiques et sociales vives " nous semble être la nécessité de prendre en compte des savoirs sociaux auxquels ces enseignements renvoient et qui s'imposent aux sujets sociaux que sont les acteurs de l'École. Cette spécificité concerne la plupart des objets d'enseignement des disciplines concernées, mais aussi d'autres objets d'enseignement qui renvoient à des questions sociales et historiques (Legardez, 2003) ou encore scientifiques, biotechnologiques (Simonneaux, 2003) ... «

\section{NOTES}

neutraliser " son enseignement sur des questions vives, en fonction du degré de vivacité perçu de ces questions. Par exemple, l'enseignant qui prépare un cours sur les revenus pourra s'appuyer sur les savoirs sociaux des élèves (sur les revenus, leur origine, leur répartition...), sur les débats entre les économistes, les sociologues, les décideurs de la politique économique, etc. L'enseignement produit pourra alors varier entre un « refroidissement » des savoirs réduits à la définition classificatoire des différents revenus (revenus primaires du travail, du capital ... et revenus de redistribution) ou un « réchauffement » par des propositions de débats argumentés sur la répartition des richesses comme question économique, sociale et politique (Legardez, socialement vives » (12).

Alain Legardez

« ADEF », Université de Provence / INRP / IUFM d'AixMarseille

(1) Ces " spécificités » sont à la convergence de caractéristiques que l'on retrouve, à des degrés divers, dans de nombreux autres cerner un domaine de recherches en didactiques des disciplines, et d'autre part de préciser les liens avec les autres domaines.

(2) A. Mercier montre néanmoins que les débats peuvent être vifs, même au sein de la discipline cible d'origine de la théorie : les mathématiques (Mercier, 2002).

(3) Notre analyse s'appuie sur les travaux des principaux auteurs de référence du champ, essentiellement ceux d'É. Chatel et de son équipe, ainsi que ceux d'E. Triby. Nos propres travaux se sont inscrits dans ce débat au sein de la didactique émergente des SES

(Beitone \& Legardez, 1995, 1997). La thèse de C. Dollo (2001) en

Transposition didactique et rapports aux savoirs

25 enseignements. Pointer ces spécificités permet donc, d'une part de 
rappelle certains arguments et propose une illustration sur l'exemple du chômage.

(4) Les SES sont « une innovation pédagogique » (Alpe, 1995) récente dans le système éducatif français, introduite à la fin des années 1960.

(5) La filière ES serait devenue la première filière « dérivative » de la filière scientifique (S), avant la filière littéraire (L) et les autres filières « relégatives ».

(6) Dans son analyse du cas des SES, Y. Chevallard (1997a) concluait déjà à un décalage entre le discours autolégitimateur d'une partie des enseignants et la réalité d'une transposition qui tend à se normaliser.

(7) Les illustrations seront choisies principalement dans le corpus des résultats de travaux récents en didactique des enseignements économiques et sociaux.

ALPE Y. (1995). - Sociologie d'une innovation pédagogique : l'enseignement des Sciences Économiques et
(8) L'enquête par questionnaire a concerné 66 professeurs de SES, 71 de STT et 84 de lycée professionnel; les entretiens complémentaires respectivement 3,3 et 4 . L'analyse a porté sur les manuels les plus usités, soit 5 de SES, 6 de STT et 6 de LP. (Voir : Legardez et al., 2002).

(9) Soit l'ensemble des acteurs qui ont une influence sur la définition des savoirs à enseigner.

(10) II faudrait y ajouter les pratiques professionnelles pour les enseignements technologiques et professionnels.

(11) Nous définissons la notion de «bonne distance » comme celle qui est construite à tout moment du processus par les acteurs de la production de savoirs-à-enseigner ... donc sans connotations en termes de valeurs ; il s'agit du résultat provisoire et instable du processus.

(12) Dans la littérature anglo-saxonne, ces questions sont souvent appelées « socio-scientific issues».

économiques et sociales au lycée. Un métier et un art. Paris : CNDP-Hachette.

\section{BIBLIOGRAPHIE}

Sociales. Thèse. Aix : Université de Provence.

ARSAC G., CHEVALLARD Y., MARTINAND J.-L., TIBERGHIEN A. (coord.) (1994). - La transposition didactique à l'épreuve. Grenoble : La Pensée Sauvage.

BEITONE A. \& LEGARDEZ A. (1995). - Enseigner les sciences économiques et sociales ; pour une approche didactique. Revue Française de Pédagogie, 112, 3345.

BEITONE A. \& LEGARDEZ A. (1997). - La monnaie et ses représentations ; éléments pour une stratégie didactique en économie. In A. Beitone \& A. Legardez (Ed.), Travaux en didactique des sciences économiques et des sciences sociales. Aix : Publications de l'Université de Provence.

CAILLOT M. \& RAISKY C. (Dir.) (1996). - Au-delà des didactiques, le didactique ; débats autour de concepts fédérateurs. Bruxelles : De Boeck.

CAZALS D. (1998). - La construction des filières dérivatives au lycée. Thèse : Toulouse.

CHARLOT B. (1997). - Du rapport au savoir, éléments pour une théorie. Paris : Anthropos.

CHARLOT B., BAUTIER É., ROCHEX J.-Y. (1992). - École et savoir dans les banlieues et ailleurs. Paris : A. Colin.

CHARLOT B., BAUTIER É., ROCHEX J.-Y. (2000). - Entre apprentissage et métier d'élève : le rapport au savoir. In A. Van Zanten (dir.), L'école, l'état des savoirs. Paris : La Découverte.

CHATEL É. et al. (1990). - Enseigner les sciences économiques et sociales, son projet, son histoire. Paris : INRP.

CHATEL É. (Dir .) (1995). - Marchés et prix. Savoirs enseignés et façons d'enseigner en sciences économiques et sociales. Paris : INRP.

CHATEL É. et al. (2001). - Elèves et professeurs en classe de SES. Pédagogies actives et activité intellectuelle de l'élève. Paris : INRP.

CHATEL É. et al. (2002). - Professeur de sciences
CHEVALLARD Y. (1991). - La transposition didactique : du savoir savant au savoir enseigné. Grenoble : La Pensée Sauvage ( $1^{\text {re }}$ éd. 1985).

CHEVALLARD Y. (1994). - Les processus de transposition didactique et leur théorisation. In G. Arsac, Y. Chevallard, J.-L. Martinand, A. Tiberghien (Coord.), La transposition didactique à l'épreuve. Grenoble : La Pensée Sauvage.

CHEVALLARD Y. (1997a). - L'enseignement des SES estil une anomalie didactique ? Skhôlé, IUFM d'AixMarseille, 6, 25-37.

CHEVALLARD Y. (1997b). - Les savoirs enseignés et leurs formes scolaires de transmission : un point de vue didactique. Skhôlé, IUFM d'Aix-Marseille, 7, 45-64.

COMBEMALE P. (Dir.) (1995). - Les sciences économiques et sociales. Paris : Hachette/CNDP.

DEVELAY M. (Dir.) (1995). - Savoirs scolaires et didactique des disciplines. Paris : ESF.

DOLLO C. (2001). - Quels déterminants pour l'évolution des savoirs scolaires en SES ? (L'exemple du chômage). Thèse, Aix : Université de Provence.

GRANGER G.-G. (1993). - La science et les sciences. Paris

JOHSUA S. (1996). - Le concept de transposition didactique n'est-il propre qu'aux mathématiques ? In $\mathrm{C}$. Raisky \& $\mathrm{M}$. Caillot (Eds.), Au-delà des didactiques, le didactique. Bruxelles : De Boeck.

JOHSUA S. (2001). - Spécificités disciplinaires, spécificités didactiques : vers une didactique comparée. In P. Venturini et al., Etudes des pratiques effectives : l'approche des didactiques. Grenoble : La Pensée sauvage.

LEGARDEZ A. (2001). - La didactique des SES ; bilan et perspectives. Aix : Publications de l'Université de Provence.

LEGARDEZ A. et al. (2002). - Circulation et reproblématisation des savoirs ; l'exemple des enseignements économiques, sociologiques, juridiques et de gestion. Rapport de recherche, Paris : INRP, 12/2002. : PUF (Coll. QSJ). 
LEGARDEZ A. (2003). - L'enseignement de questions sociales et historiques, socialement vives. Lausanne : Le Cartable de Clio, 3, 245-253.

LEGARDEZ A. (2004). - Enseigner l'économie : une perspective didactique. In A. Diemer (Éd.), Enseigner l'économie. Paris : L'Harmattan, 151-168.

LEGARDEZ A. (2004). - Savoirs des élèves et débat argumenté. L'exemple de l'incertitude et du risque en économie. In M. Tozzi (Dir.), La discussion dans l'enseignement et la formation. Montpellier : Les Cahiers du Cerfee, 19 (2003), 89-99.

LEGARDEZ A. \& LEBATTEUX N. (2002). - Enseignement de l'entreprise et représentations sociales en lycée professionnel tertiaire. Aster, 34, 181-211.

LEZIART J. (1995). - Le métier de lycéen et d'étudiant ; rapport aux savoirs et réussite scolaire. Paris : L'Harmattan.

MARTINAND J.-L. (1986). - Connaître et transformer la matière. Berne : Peter Lang.

MARTINAND J.-L. (2001). - Pratiques de référence et problématique de la référence curriculaire. In A. Terrisse (Éd.), Didactique des disciplines. Les références au savoir. Bruxelles : De Boeck.

MERCIER A. (2002). - La transposition des objets d'enseignement et la définition de l'espace didactique, en mathématiques. Revue Française de Pédagogie, 141, 135-171.

MERCIER A., SCHUBAUER-LÉONI M.-L., SENSEVY G. (2002). - Vers une didactique comparée. Revue Française de Pédagogie, 141, 5-16.

PETITJEAN A. (Ed.) (1998). - La transposition didactique en français. Pratiques, 97-98.

SIMONNEAUX L. (2003). - Argumenter dans les débats en classe sur une technoscience controversée. Aster, 37, 189-214.

TERRISSE A. (Ed.) (2001). - Didactique des disciplines. Les références aux savoirs. Bruxelles : De Boeck.

TRIBY E. (1995). - Les SES sont-elles une discipline ? L'interrogation de la didactique. In P. Combemale (Dir.), Les sciences économiques et sociales. Paris : Hachette-CNDP.

TRIBY E. (1997). - Enseigner l'économie par les représentations des élèves : le cours sur la monnaie. In A. Beitone \& A. Legardez (Ed.), Travaux en didactique des sciences économiques et des sciences sociales. Aix : Publications de l'Université de Provence.

VERGES P. (1991). - Les représentations sociales de l'économie : une forme de connaissance. In D. Jodelet, Les représentations sociales. Paris : PUF ( $1^{\text {re }}$ éd., 1989). 